\title{
STUDYING THE EFFECT OF AN ADDITIONAL ACTIVE RESISTANCE IN THE FIELD WINDING CIRCUIT ON STARTING CHARACTERISTICS OF SALIENT-POLE SYNCHRONOUS MOTORS
}

\begin{abstract}
On the basis of the developed method for calculating steady-state asynchronous operation modes of salient-pole synchronous motors, a procedure of mathematical modelling of the starting characteristics is proposed. The problem of calculating the steady-state asynchronous mode is solved as a boundary value one for differential equations of motor circuit electrical equilibrium. Algebraization of the system of differential equations is carried out by approximating the equations of state using cubic spline functions on a grid of period nodes, taking into account the periodic law of variation of the coordinates. This results in the changeover from continual values to nodal ones. The starting static characteristics are calculated using the parameter continuation method. The study of the effect of the starting resistance value on the asynchronous characteristics of the motor relied on a mathematical model of the motor taking into consideration real field circuits, saturation and asymmetry of the magnetic path. References 10, figures 5.

Key words: salient-pole synchronous motor, asynchronous operation mode, starting resistance, static characteristics, boundary value problem, spline approximation.
\end{abstract}

На основе разработанного метода расчета установившихся асинхронных режимов явнополюсных синхронных двигателей предложена методика математического моделирования пусковых характеристик. Задача расчета стационарного асинхронного режима решается как краевая для дифференциальных уравнений электрического равновесия контуров двигателя. Алгебраизация системы дифференциальных уравнений осуществляется путем аппроксимации уравнений состояния кубическими сплайн-функциями на сетке узловых точек периода с учетом периодического закона изменений координат. В результате осуществляется переход от их континуальных значений к узловым. Расчет пусковых статических характеристик осуществляется методом продолжения по параметру. Для исследования влияния величины пускового сопротивления на асинхронные характеристики двигателя использовано математическую модель двигателя, в которой учитываются реальные контуры пусковой обмотки, насыщение и несимметрия магнитопровода. Библ. 10, рис. 5.

Ключевые слова: явнополюсный синхронный двигатель, асинхронный пуск, пусковое сопротивление, статические характеристики, краевая задача, аппроксимация сплайнами.

Introduction. The use of synchronous motors (SMs) for high-power electric drives provides high technical and economic indicators, but there is a problem of their startup [1-3]. SMs are manufactured mostly with salient poles, on which an excitation winding is located. To access the synchronous mode, it is necessary to accelerate the rotor of the motor to close to the synchronous speed of rotation, after which to supply direct current to the winding excitation, causing the rotor to enter the synchronization with the magnetic field of the stator winding.

The problem of starting synchronous motors, which operate in powerful electric drives, is one of the main [4-6]. This problem is especially relevant for those synchronous electric drives, in which the start is carried out under load. Its essence is to provide the necessary starting torque, which is conditioned by the operating conditions of the drive. In practice, there are various methods of start-up and circuits that implement them, which are characterized by disadvantages and advantages. The choice of a particular method for solving the problem of start-up requires effective in relation to the adequacy methods for obtaining functional dependencies that would enable to analyze both qualitative and quantitative aspects of the dynamics of the starting process. They are the basis for the development of starting systems [4], which ensure the technological reliability of electrical equipment and its cost-effectiveness for electricity consumption, which is important for electric drives, which operate under frequent start-ups.
As is known, the main method of starting a SM is asynchronous start, which is carried out by its direct switching on the nominal voltage, and the electromagnetic torque develops due to the position of the starter winding located at the poles of a salient rotor [2]. Generally, the excitation winding is closed at the start time on a discharge active resistance with a value that is (5-10) times more than its own resistance $r_{f}$, less frequently short-circuit. The purpose of this resistance is to avoid overvoltage in the excitation winding at the beginning of the asynchronous start-up. The problem of the effect of an additional starting resistance in the excitation winding on the value of electromagnetic torque was generally not considered or investigated using so simplified mathematical models that reliable results are unlikely.

The electromagnetic torque, created in the asynchronous mode by the starting winding of the SM rotor, lacking for its start-up under the load, therefore different techniques for its increase are used [5, 6]. For example, one of ways to solve the problem of increase the SM starting characteristics is serial connection of capacitors to the excitation windings which compensate its inductive resistance, but of the wrong choice of their capacities can call result in the phenomena of the voltage resonance or to decrease the starting electromagnet torque instead of its increase [7]. These disadvantages are enhanced by the way to improve the startup characteristics by using active resistance in the excitation winding [1]. However, the problem of completing an

(C) V.S. Malyar, A.V. Malyar 
asynchronous start-up of SM, its excitation winding is shorted to an active resistance, despite of its importance for practice, is still not solved till now.

The problem of the reasonable choice of the value of the additional resistance in the field winding can be solved by the way of mathematical modeling, but it requires utilization of the improved SM mathematical models and the development of effective numerical analysis techniques.

The goal of the paper is development of a mathematical model and technique of calculation of asynchronous modes of SM in order to study the influence of active resistance in the circuit of excitation winding on the starting characteristics of SM.

A mathematical model. We consider the SM, whose stator winding is powered by a three-phase network, and on the rotor is a starter winding with $n$ rods at each pole and an excitation winding.

As is known [2], the stable synchronous modes of SM can be analyzed on the basis of the use of equivalent circuits, but under the conditions of non-synchronous rotation of the rotor, even in steady state, the electromagnetic processes occurring in the electric circuits of the motor are dynamic. And since equivalent circuits do not allow to take into account the main factors influencing the dynamics of processes in the SM, they are not suitable for analysis of asynchronous modes of the SM.

Due to the salient pole construction of the rotor and the asymmetry of the starting winding, the distribution of the magnetic flux density in the air gap differs from the sinusoidal, and the electromagnetic torque in the asynchronous mode has a constant component and a variable one that fluctuates with a double frequency. In addition, as a result of saturation of the SM magnetic circuit, the flux linkages of the circuits, and hence electromagnetic parameters, are nonlinearly dependent on all its currents.

The practice of calculating processes in SM shows that the initial determining influence on the behavior of the motor in asynchronous mode is due to a starting winding, and therefore the accuracy of the results of the calculation depends on the adequacy of its description in the mathematical model. Other factors are the nonsinusoidal distribution of the magnetizing forces of the electrical circuits of the SM and the saturation of the magnetic system. Only taking into account these factors in their interconnection can serve as the basis for the development of appropriate calculation methods based on the use of modern numerical methods.

Asynchronous modes of the SM are described by the nonlinear system of differential equations (DE) of the electromechanical equilibrium, which can be written in real phase or in transformed coordinate systems. The choice of the coordinate system is determinative because a possibility to take into account factors influencing the dynamics of processes depends on it. In addition, for a salient-pole SM the technique of the starting winding modeling is important: as two equivalent or real physical circuits.

Most of the analysis tasks do not require the use of mathematical modeling of processes in each rod of the starting winding, so it can be equivalented with two orthogonal circuits oriented on the longitudinal $(d)$ and transverse $(q)$ axes [2]. Since in creating an electromagnetic torque in asynchronous mode the starting winding is decisive, in order to obtain adequate results, we consider the real circuits formed by its rods [10], between which there are nonlinear mutual inductive connections due to the saturation of the magnetic core. However, the winding of a stator, since it is symmetric and is fed by a symmetric three-phase voltage system, can be considered in the known $d, q$ coordinate axes. Under these conditions, the process of asynchronous start-up of the SM in the case of closing the excitation winding to the active resistance $r_{a}=k \cdot r_{f}$, where $k$ is the multiplicity of the additional resistance, is described by the nonlinear system of DE of electromagnetic equilibrium of the circuits

$$
\begin{gathered}
\frac{d \psi_{d}}{d t}=\omega \psi_{q}-r i_{d}+U_{m} \sin \theta ; \\
\frac{d \psi_{q}}{d t}=-\omega \psi_{d}-r i_{q}+U_{m} \cos \theta ; \\
\frac{d \psi_{1}}{d t}=-r_{1,1} i_{1}+r_{1,2} i_{2}-r_{1, n} i_{n} ; \\
\frac{d \psi_{n}}{d t}=-r_{n, 1} i_{1}+r_{n, n-1} i_{n-1}-r_{n, n} i_{n} ; \\
\frac{d \psi_{f}}{d t}=-\left(r_{f}+r_{a}\right) i_{f},
\end{gathered}
$$

where $\psi_{k}, i_{k}(k=d, q, 1, \ldots, n, f)$ are the flux linkages and currents of the corresponding circuits; $r, r_{f}, r_{j k}$ are the active resistances of windings: of the stator, field and damping circuits $\left(r_{j k}\right)$, intrinsic - at $j=k$ and mutual - at $j \neq k ; \theta$ is the angle of rotor runoff.

The DEsystem (1), supplemented by the equation of rotor motion, allows us to investigate the transients in asynchronous modes of operation of the SM. Obviously, the transient asynchronous startup requires a numerical integration of the DE in a time interval sufficient for the motor to output to the asynchronous mode. However, for the design of the SM and, in particular, the corresponding starting systems for electric drives [4], and the study of their starting properties, it is necessary to have an effective algorithm for calculating static characteristics, which would enable to investigate the influence of the starting winding and excitation winding parameters on the processes in the SM in the starting mode.

As is well known [2], in the asynchronous mode of the salient-pole SM, the angle $\theta$ the rotor runoff is continuously increasing by law

$$
\theta=-\omega_{0} \int s d t+\theta_{0}
$$

where $\theta_{0}$ is its initial value, $s=1,0-\omega / \omega_{0}, \omega_{0}, \omega$ are the angular speed of the voltage power and angular rotor speed of the motor, expressed in electric radians.

Since with unbounded growth of the angle $\theta$ the functions $\sin \theta$ and $\cos \theta$, which determine the value of the applied voltage, are changed according to the periodic law, then the flux linkage, the circuit currents and, as a consequence, the electromagnetic torque, are periodic 
functions. The sliding $s$ of the rotor within the period fluctuates relative to the mean value

$$
s_{0}=\frac{1}{T} \int_{0}^{T_{a}} s d t
$$

however, for solving most tasks it can be accepted unchanged and equal to the average value.

A technique of problem solution. Investigation of the influence of the active resistance in the circuit of the excitation winding on the electromagnetic torque can be made on the basis of the calculation of the static characteristic, which is obtained [2] by calculating the sequence of stable asynchronous modes, which are calculated for different values of sliding. The lawfulness of such an approach is due to the fact that the mechanical time constant is far greater than electromagnetic. Since in the stable asynchronous mode with sliding $s$ processes in the SM are changed with the period

$$
T_{a}=2 \pi /\left(s \omega_{0}\right)
$$

then the task of its calculation is to determine the periodic dependencies of the coordinates. Their calculation by solving an evolutionary problem requires the integration of the DE system in a significant time interval, and therefore not suitable for the calculation of static characteristics. Calculate these dependencies with the minimum computations can be by solving the boundary value problem for the DE system (1) with periodic boundary conditions [9].

In order to reduce the description of the calculation algorithm for the periodic dependencies of the coordinates of the mode on the $T_{a}$ period, we write the DE system (1) by one vector equation

$$
\frac{d \vec{\psi}}{d t}=\vec{z}(\vec{\psi}, \vec{i}, \vec{u}, t),
$$

where

$$
\vec{z}=\begin{array}{|c|}
\hline U_{m} \sin \theta+\omega_{0}(1-s) \psi_{q}-r i_{d} \\
\hline U_{m} \cos \theta-\omega_{0}(1-s) \psi_{d}-r i_{q} \\
\hline-r_{11} i_{1}+r_{12} i_{2}-r_{1 n} i_{n} \\
\hline \vdots \\
\hline-r_{n 1} i_{1}+r_{n, n-1} i_{n-1}-r_{n n} i_{n} \\
\hline-\left(r_{f}+r_{a}\right) i_{f} \\
\hline
\end{array}
$$

The equation (2) includes the time coordinate $t$, and therefore, calculating with its use of static characteristics is a problem. However, in the condition of constant sliding, it describes the stationary asynchronous mode of the SM, which is characterized by a periodic nonharmonic change in coordinates. Due to the nonlinear electromagnetic connections between the motor circuits, the DE system (2) describing electromagnetic processes can not be reduced to algebraic ones by the transition to a complex variable, and the calculation of the steady state is to determine the functional dependencies of instantaneous coordinate values during the $T_{a}$ period.

Determining these dependencies is a boundary value problem for the DE system (2). In mathematics, as a rule, boundary-value problems are considered for second-order DEs, but due to the fact that boundary conditions are given by periodic dependencies, their definition is possible for first order DEs. To solve the boundary-value problem, the DE system (2) needs to be algebratized on a period, that is, to make a transition from differential equations to algebraic ones.

Different methods of algebrization are known in the literature, among which are the difference methods of various orders, collocation, including trigonometric, and others. However, projective methods [8] proved most effective, the essence of which is that the solution of the operator equation in a given subspace is determined by projecting a differential equation on some other subspace. Projection methods are the basis for constructing various computational schemes for solving boundary value problems [8]. The difference between them is to choose basic functions for approximation of the corresponding curves. In the paper the method of analysis of asynchronous modes of SM operation is proposed by the projection method developed on the basis of the use of cubic splines [9]. The essence of the method is the transition from the DE system (2) of a continuous variable to discrete algebraic equations. For this we divide the period $T$ into $m$ intervals $h_{j}=t_{j}-t_{j-1}(j=\overline{1, m})$ and on each of them the components of the vector $\vec{\psi}$ are approximated by splines of the third order of the form

$$
\psi(t)=a_{j}+b_{j}\left(t_{j}-t\right)+c_{j}\left(t_{j}-t\right)^{2}+d_{j}\left(t_{j}-t\right)^{3},
$$

where $a_{j}, b_{j}, c_{j}, d_{j}$ are the spline coefficients, the relations between which are determined by the properties of the spline functions, which in the nodes of the mesh are linked together by the value of functions and are twice differentiated throughout the period. The relationships between the spline coefficients follow from its properties and are determined for each coordinate of the vector $\vec{\psi}$ only by the mesh of nodes in the period

$$
\begin{aligned}
& \frac{3}{h_{j}} a_{j-1}-\left(\frac{3}{h_{j}}+\frac{3}{h_{j+1}}\right) a_{j}+\frac{3}{h_{j+1}} a_{j+1}= \\
& =h_{j} c_{j-1}+2\left(h_{j}+h_{j+1}\right) c_{j}+h_{j+1} c_{j+1} . \\
& b_{j}=\frac{a_{j-1}-a_{j}}{h_{j}}-\frac{h_{j}}{3}\left(c_{j-1}-2 c_{j}\right) .
\end{aligned}
$$

In the matrix form equation (4), taking into account the conditions of the periodicity of the spline $a_{n+j}=a_{j}$, $c_{n+j}=c_{j}$, has a form

$$
W_{21} \vec{A}+W_{22} \vec{C}=0,
$$

where $\vec{A}=\left(a_{1}, \ldots, a_{N}\right)^{*} ; \vec{C}=\left(c_{1}, \ldots, c_{N}\right)^{*}$, and the upper index $\left(^{*}\right)$ means the transposition of the vector.

Equation (2) for the $j$-th node of the mesh has a form

$$
\left.\frac{d \vec{\psi}}{d t}\right|_{j}=\vec{z}_{j}\left(\vec{\psi}_{j}, \vec{i}_{j}, \vec{u}_{j}\right),
$$

in which $\vec{u}_{j}=\left(U_{m} \sin \theta_{j}, U_{m} \cos \theta_{j}, 0, \ldots, 0\right)^{*}$.

Because, as it follows from (3), in the $j$-th node

$$
a_{j}=y_{j} ; \quad b_{j}=-d y /\left.d t\right|_{j}=-\vec{z}_{j},
$$

equation (5) has a form

$$
\frac{a_{j-1}-a_{j}}{h_{j}}-\frac{h_{j}}{3}\left(c_{j-1}-2 c_{j}\right)=-z_{j}
$$


or in the matrix form

$$
W_{11} \vec{A}+W_{12} \vec{C}=-\vec{Z}
$$

where $\vec{Z}=\left(z_{1}, \ldots, z_{m}\right)^{*}$. Consequently, the problem of determining the spline (3) is reduced to the determination of the coefficients $a_{j}$ and $c_{j}$.

Defining a vector $\vec{C}$ from equation (6), we obtain the equation for determining the nodal values of the coordinates (vector $\vec{A}$ )

$$
\left(W_{11}+W_{12} W_{22}^{-1} W_{21}\right) \vec{A}=-\vec{Z}
$$

where $W_{11}, W_{12}, W_{21}, W_{22}$ are the block-matrices of size $m \times m$, the elements of which are determined exclusively by the coefficients of the equations (4), (5).

The obtained system (9) of the algebraic equations of the $k m$ order, where $k=2+m+1$ is the number of equations of the DE system DR, is a discrete reflection, which approximates it on the mesh of nodes of the period $T_{a}$ of the repetition of the process. It includes coordinate values that correspond to fixed values of the time coordinate $t_{j}$ in the nodes of the period, the relationships between which at each time point are nonlinear. Because $\vec{A}=\vec{\psi}$, a $\vec{Z}=\vec{Z}(\vec{\psi}, \vec{u})$, and, moreover, $\vec{\psi}=\vec{\psi}(\vec{i})$. it can be presented as a nonlinear equation of the form

$$
\vec{Q}(\vec{\Psi}, \vec{I}, \vec{U})=0 \text {. }
$$

Its solution is the values of the vector

$$
\vec{I}=\left(\vec{i}_{1}, \ldots, \vec{i}_{j}, \ldots, \vec{i}_{m}\right)^{*}, \quad \vec{i}_{j}=\left(i_{d j}, i_{q j}, i_{1 j}, \ldots, i_{n j}, i_{f j}\right)^{*},
$$

which are determined by the vector of applied voltages and electromagnetic parameters of SM.

For the solution of the nonlinear algebraic system (10), the method of continuation by the parameter [8,9] in combination with the iterative method of Newton is applied, according to which on each $l$-th step iteration the vector $\vec{I}$ is corrected by the formulas

$$
\vec{I}^{(l+1)}=\vec{I}^{(l)}-\Delta \vec{I}^{(l)} ; \quad W \Delta \vec{I}^{(l)}=\vec{Q}^{(l)},
$$

where $\vec{Q}^{(l)}$ is the vector of the discrepancy of the system (10) at $\vec{I}=\vec{I}^{(l)}$.

The inducing force in equation (10) is the vector $\vec{U}=\left(\vec{u}_{1}, \ldots, \vec{u}_{m}\right)^{*}$, increasing which from zero to nominal value, we obtain the values of vectors $\vec{\Psi}$ and $\vec{I}$ that correspond to the specified value of sliding $s=s_{0}$. The obtained coordinate values are the initial conditions for the calculation of static characteristics.

Elements of the blocks of the Jacobi matrix are differential inductive resistances of the motor circuits which are determined by calculating the equivalent circuit of the magnetic circuit of the SM in accordance with the accepted model [10]. The result of the calculation of the magnetic circuit is the determination of the magnetic flux density curve in the air gap of the motor by the theory of circuits, which enables to determine the flux linkages of the circuits that belong to the vector of discrepancies and depend on the set of currents of all motor circuits.

To study the effect of the value of the additional active resistance in the winding of excitation on the starting characteristics it is necessary, by setting the value of sliding, according to the above algorithm, to determine the value of coordinates of the mode with the value of the starting resistance in the excitation winding $r_{a}=0$, and then to increase its value within the required limits, correcting the discrepancy by the Newton method. The set of coordinates defined at each step of integration, allows us to determine the multidimensional characteristic as their dependence on the starting resistance in the excitation winding.

Similarly, the calculation of static starting characteristics as coordinate dependencies on sliding is performed. In this case, the additional resistance in the excitation winding is assumed unchanged, and sliding varies within the specified range.

To study the transient process of starting a SM, it is necessary together with the DE system (1) to integrate by a numerical method the equation of the mechanical equilibrium of the rotor

$$
\begin{gathered}
\frac{d \theta}{d t}=\frac{\omega}{\omega_{0}}-1 \\
\frac{d \omega}{d t}=\frac{p_{0}}{J}\left(\frac{3}{2} p_{0}\left(\psi_{d} i_{q}-\psi_{q} i_{d}\right)-M_{c}\right),
\end{gathered}
$$

where $p_{0}$ is the number of pairs of poles; $J$ is the moment of inertia of the electric drive system; $M_{c}$ is the moment of loading on the motor shaft.

At each step of integration to determine the electromagnetic parameters, which are the elements of the matrix $\partial \vec{\psi} / \partial \vec{i}$ of differential inductances of the motor circuits, it is necessary to solve a system of equations that describes the branched magnetic circuit of the SM.

Note that when calculating the periodic change of coordinates by the method of solving a boundary value problem, the magnetic state is calculated only for node points of the period, which is much smaller than their calculation by the method of setting.

Results of investigations. Below are examples of calculation results based on the developed method of stable modes and static characteristics of salient-pole synchronous motors СДС3-17-41-16 $(P=1600 \mathrm{~W}$, $U=6000 \mathrm{~V}, 2 p=16 ; n=8)$ and СДНЗ-2-19-49-24 $(P=1600 \mathrm{~kW}, U=6000 \mathrm{~V}, 2 p=24 ; n=5)$, which differ in the number of pairs of poles and rods at the pole.

An example of the periodic dependencies of the excitation current and the electromagnetic torque of the СДС3 motor at sliding $s=1.0$ is shown in Fig. 1 .

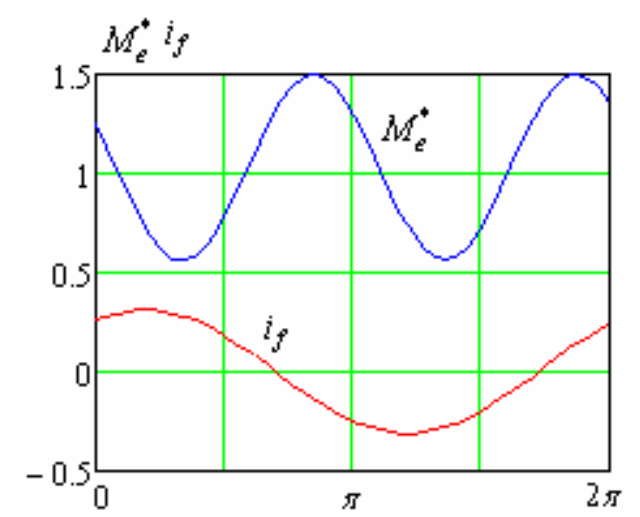

Fig. 1. Periodic current dependencies in the excitation winding of the СДСЗ motor and the electromagnetic torque with a short excitation winding 
As can be seen from Fig. 2, the dependence of the starting $(s=1)$ electromagnetic torque on the additional resistance in the excitation winding for the SM of the same power, but with different number of pairs of poles and rods on each of them significantly differ. The calculation of the dependence of the propulsion electromagnetic torque on the starting resistance allows one to determine its value, at which the maximum torque will be.

Analysis of the calculation results (Fig. 2) shows that the 5-10-time value of the starting resistance, which is usually used, is not optimal in terms of providing the maximum value of the starting torque. However, a significant overstatement of the starting resistance can give a reverse effect.
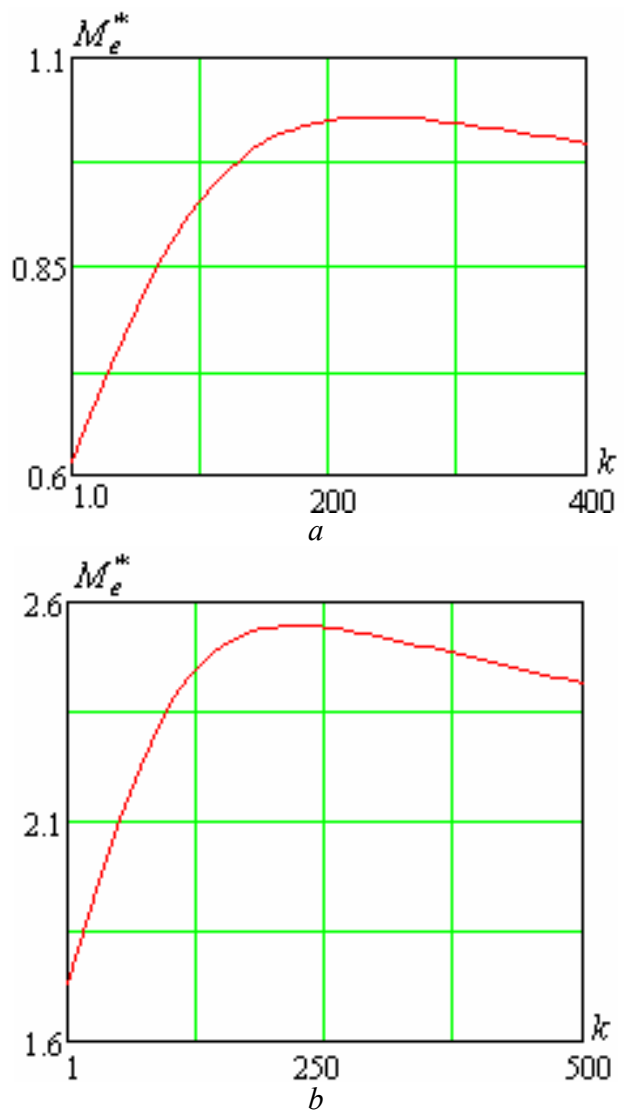

Fig. 2. Dependencies of the starting $(s=1)$ electromagnetic torque on the multiplicity of the additional resistance in the excitation winding for the motors СДСЗ $(a)$ and СДНЗ $(b)$

The developed mathematical model and calculation technique allows to investigate the effect on the asynchronous characteristics of the starting winding and excitation winding separately, which is important both for the design of the starting winding, and the development of optimal systems for automated control of the starting of the SM. To do this, in the program of calculating, the resistance of the winding, which must be excluded from consideration, is multiplied by a large $\left(10^{3}-10^{6}\right)$ number. An example of the results of such studies is shown in Fig. 3, of which it is evident how different the created by the field winding asynchronous moments for different motors. In particular, at the first moment of the asynchronous start-up the asynchronous torque generated by the excitation winding, has little effect on the electromagnetic torque as a whole. Its maximum value is manifested in small sliding, but it is, as can be seen from Fig. 3, is significantly different for different motors.

In the absence of a starting winding in the vicinity of the sliding value $s=0.5$ in the curve of the asynchronous moment, the uniaxial effect is manifested (curve in Fig. 4,b). However, due to the salient polarity of the SM rotor, the failure in the curve of the electromagnetic torque is practically leveled by the starting winding, although for a sufficiently large value of the starting resistance in the excitation winding it manifests itself (Fig. 5). The corresponding choice of the value of the starting resistance can achieve not only increasing the starting torque but also maximizing it (Fig. 5). In addition, at the same time, the starting current in the stator winding decreases somewhat.
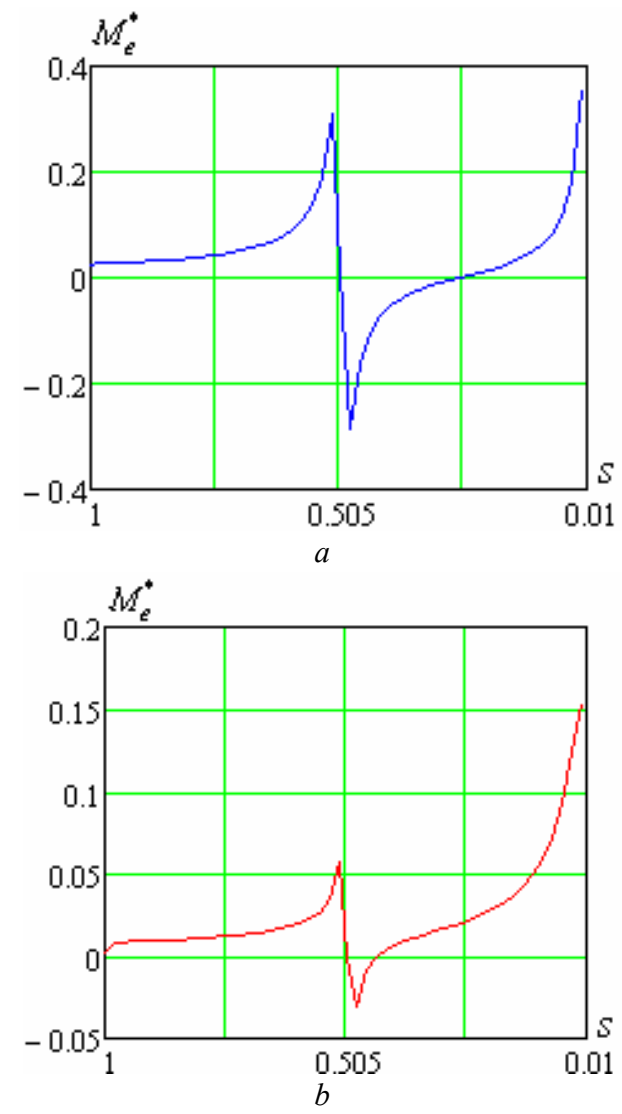

Fig. 3. Starting characteristics of the electromagnetic torque that is created by an excitation winding in the absence of a starting winding for motors СДСЗ $(a)$ and СДНЗ $(b)$

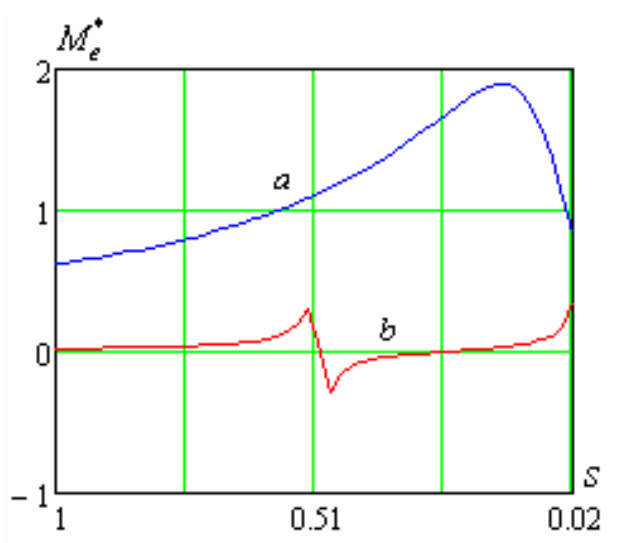

Fig. 4. Mechanical characteristics of the motor СДС3: $a$ - with a shorted excitation winding and a starting winding; $b$ - in the absence of a starting winding 


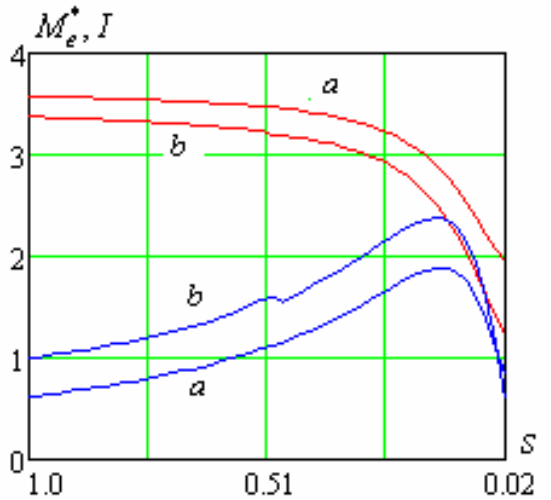

Fig. 5. Dependencies of the electromagnetic torque and the current of the motor СДС 3 on the sliding with shorted $\left(r_{a}=0\right)$ excitation winding $-(a)$ and closed on the additional resistance $r_{a}=250 r_{f}-(b)$.

\section{Conclusions.}

1. A mathematical model and technique of calculation of asynchronous modes and static characteristics of salient-pole synchronous motors are developed, which makes it possible to investigate the influence of the value of the starting active resistance in the excitation winding on the value of the electromagnetic torque.

2 . It is shown that by connection of the resistors in the excitation winding, it is possible not only to increase the driving electromagnetic torque of the motor, but also to influence its starting characteristics in general.

3 . The developed mathematical model and calculation technique can be used in the process of designing salientpole synchronous motors for optimization of their starting resistance and starting winding in order to form the starting characteristics required for a specific electric drive.

\section{REFERENCES}

1. Abramovich B.N. About starting resistance ratio of synchronous motors. Electrical engineering industry. Electric machines, 1974, no.4(38), pp. 14-15. (Rus).

2. Voldek A.I., Popov V.V. Elektricheskiye mashiny. Mashiny peremennogo toka [Electric machines. AC machines]. Saint Petersburg, Piter Publ., 2010. 350 p. (Rus).
3. Baskov S.N., Radionov A.A., Usatyi D.Yu. Start of asynchronous motors in electric drives with increased starting torque. Russian Electromechanics, 2004, no.2, pp. 47-49. (Rus.) 4. Chornyy A.P., Gladyr A.I., Osadchuk Yu.G., Kurbanov I.R., Voshun A.N. Puskovyje sistemy nereguliruemykh elektroprivodov [Start-up systems of unregulated electric drives]. Kremenchug, Scherbatykh A.V. Publ., 2006. 280 p. (Rus).

5. Pivnyak G.G, Kirichenko V.I, Boroday V.A. About new direction in improvement large synchronous electric motors. Technical electrodynamics. Thematic issue «Problems of modern electrical engineering», 2002, chapter 2, pp. 62-65. (Rus).

6. Kirichenko V.I., Boroday V.A., Yalanskiy A.A. Improved properties of starting modes of large synchronous machines. Visnik Of The Volodymyr Dahl East Ukrainian National University, 2003, no.6(64), pp. 38-40. (Rus).

7. Malyar V.S., Malyar A.V., Dobushovska I.A. Simulation of asynchronous modes of synchronous motors with capacitors in the excitation circuit. Electrical engineering \& electromechanics, 2012, no.5, pp. 31-33. (Ukr). doi: 10/20998/2074-272X.2012.5.06.

8. Ortega J., Poole W. An introduction to numerical methods for differential equations. Boston, Jr. Pitman Publ. Inc., 1981. $344 \mathrm{p}$.

9. Malyar V.S, Malyar A.V. Mathematical simulation of periodic modes of electrotechnical appliances. Electronic modeling, 2005, vol.27, no.3, pp. 39-53. (Rus).

10. Filts R.V, Lyabuk N.N. Matematicheskoye modelirovaniye yavnopolyusnykh sinkhronnykh mashin [Mathematical simulation of salient-pole synchronous motor]. Lviv, Svit Publ., 1991. 176 p. (Rus).

\section{Received 06.01.2018}

V.S. Malyar ${ }^{1}$, Doctor of Technical Science, Professor, A.V. Malyar ${ }^{1}$, Doctor of Technical Science, Professor,

${ }^{1}$ Lviv Polytechnic National University,

12, S. Bandera Str., Lviv, 79013, Ukraine, phone +380322582119 ,

e-mail: mvs@polynet.lviv.ua; svmalyar@polynet.lviv.ua

How to cite this article:

Malyar V.S., Malyar A.V. Studying the effect of an additional active resistance in the field winding circuit on starting characteristics of salient-pole synchronous motors. Electrical engineering \& electromechanics, 2018, no.2, pp. 18-23.

doi: 10.20998/2074-272X.2018.2.03. 